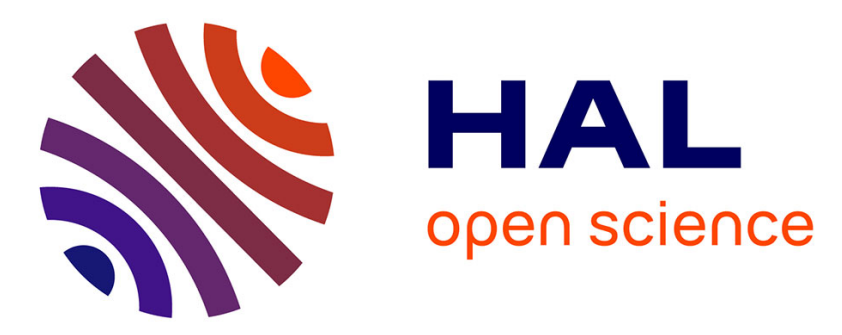

\title{
Local damage evaluation of a laminate composite plate using ultrasonic birefringence of shear wave
}

\author{
Sandrine T. Rakotonarivo, C. Payan, J. Moysan, C. Hochard
}

\section{To cite this version:}

Sandrine T. Rakotonarivo, C. Payan, J. Moysan, C. Hochard. Local damage evaluation of a laminate composite plate using ultrasonic birefringence of shear wave. Composites Part B: Engineering, 2018, 142, pp.287-292. hal-02043375

\section{HAL Id: hal-02043375 https://hal.science/hal-02043375}

Submitted on 21 Feb 2019

HAL is a multi-disciplinary open access archive for the deposit and dissemination of scientific research documents, whether they are published or not. The documents may come from teaching and research institutions in France or abroad, or from public or private research centers.
L'archive ouverte pluridisciplinaire $\mathbf{H A L}$, est destinée au dépôt et à la diffusion de documents scientifiques de niveau recherche, publiés ou non, émanant des établissements d'enseignement et de recherche français ou étrangers, des laboratoires publics ou privés. 


\title{
Local damage evaluation of a laminate composite plate using ultrasonic birefringence of shear wave
}

\author{
S. T. Rakotonarivo*, C. Payan, J. Moysan, C. Hochard \\ Aix Marseille Univ, CNRS, Centrale Marseille, LMA, Marseille, France
}

\begin{abstract}
This paper presents an ultrasonic non-destructive testing method to locally characterize composite material damage through the analysis of the anisotropy behavior variation induced by damage. The approach is based on shear wave contact measurements and analyses shear wave birefringence to estimate variation of the attenuation coefficient and shear moduli ratio between warp and weft directions. The method only requires a single piezo-electric contact sensor and thus, is easy to implement for practical applications. Moreover, unlike other methods, it does not require any reference measurement in a calibrated medium such as water to evaluate attenuation coefficient variation and shear moduli ratio. It is shown that the proposed method allows to locally qualify damage with good repeatability and robustness.

Keywords: Non-destructive testing, Ultrasonics, Damage induced anisotropy, Laminates, Shear wave birefringence
\end{abstract}

\section{Introduction}

Composite materials are widely used in many sectors of activity such as aeronautics, automotive and naval industries, or civil engineering that require the use of both lightweight and mechanically efficient materials. In order to ensure 5 integrity and safety of any composite structure, it is primordial to conduct mate-

\footnotetext{
* Corresponding author

Email address: sandrine.rakotonarivo@univ-amu.com (S. T. Rakotonarivo )
} 
rial testing throughout the structure life from the manufacturing process to the in service phase. Non-destructive testing techniques based on ultrasounds have shown great potential throughout the last decades to reach this goal. Indeed ultrasonic waves allow to achieve local or global composite structure inspections to evaluate the structure health and determine if it is damaged [1, 2, 3].

When a composite structure gets damaged, a loss of stiffness is generally observed with respect to a pristine structure [4, 5]. This loss of stiffness, also called damaged factor, allows to qualify the material damage at the macroscopic scale. In order to get an estimate of this factor, one needs to measure first the elasticity constants of the composite and follows their evolution. One approach to measure elasticity properties of the composite consists in measuring ultrasound velocity of bulk waves on plates immersed in water and for many incident directions [1, 6, 7, 8, 9. However, those approaches based on immersed ultrasound measurements are difficult to implement for practical applications.

${ }_{20}$ The Impulse Excitation Technique was recently applied to damaged composite laminates for measuring the residual elastic properties [10. As it requires two side access, in situ inspection of structures with the later technique remains challenging. Other methods exploit Lamb waves dispersion curves for characterizing composite plates from laser measurements [1] or from air-coupled 25 ultrasonic transducers requiring a single plate side access [12]. An other category of approaches utilizes acoustic emission techniques to monitor composite structure and to detect damage mechanisms by detecting the released energy due to these events in a structure [13, 14].

Alternatively, it was shown that acoustic birefringence provides information about the stiffness of composite plates and is sensitive to damage [15, 16]. The approach is based on the analysis of the anisotropy behavior of orthotropic composite plates. Acoustic birefringence represents difference in the ultrasonic velocities of two orthogonally polarized shear waves propagating through the thickness of a plate and polarized into both principal perpendicular directions 35 to their average value. It is sensitive to anisotropy in the microstructure (texture, fibre alignment) and stress [17]. Hence, analysis of acoustic birefringence 
through measurements of shear wave velocity in various directions of polarization provide information about the direction of stress [18] or the fibre orientation [15, 19. In addition to the primary anisotropy of the pristine material, the damage modifies its mechanical properties (loss of stiffness) and therefore its anisotropy according to the encountered sollicitations. The material loss of stiffness modifies the ultrasonic shear wave velocities and thus the shear wave birefringence behavior of the material. Ultrasonic shear wave birefringence is then correlated to the material damage. In particular, it was shown that birefringence has a good potential for characterizing damage on composite laminates [15. 16]. In this paper, this approach is extended to locally evaluate damage on a laminate plate through estimation of shear moduli ratio and attenuation coefficient variation from ultrasound contact pulse-echo measurements. Using the experimental procedure proposed in [15, 16, 20] for shear wave birefringence analysis, a new method of estimation of the ultrasound parameters is proposed for characterizing damage. In the presented approach, the inversion algorithm exploits the whole frequency content of the signal and applies a global optimization scheme for estimating the shear moduli ratio and the attenuation coefficient variation. Moreover, reference measurements in a calibrated medium is no more needed for estimation of the attenuation parameter as in 20]. Experimental robustness/accuracy of the method is tested by performing many measurements at different day time. The method successfully manages to locally characterize damage on a unbalanced woven composite plate made of fiber glass reinforcement in an epoxy matrix. Interest for woven ply laminates relies on their better resistance to delamination than unidirectional ply and their low sensitivity to transverse rupture [21].

Theoretical basis of the method are presented in section 2. Then, section 3 presents the experimental tests conducted on pristine and damaged samples. Results are discussed in the following paragraph. Conclusions are presented in 65 section 5 . 


\section{Damage evaluation from shear wave birefringence analysis}

This section presents the shear wave birefringence theory with general formulations for layered composites made of orthotropic plies with any orientation between plies. Then, simplified expressions are derived for a composite made of plies with identical orientation and properties. Further, the method for measuring off plan shear moduli and attenuation variation for characterizing the material is presented in section 2.2 .

\subsection{Theory of shear wave birefringence}

The approach is based on the analysis of shear wave phase and amplitude variations versus polarization angle from contact pulse-echo measurements following the experimental set-up depicted in Fig. 1. Fiber-reinforced composites have mechanical properties varying according to manufacturing process. In consequence, due to their anisotropic nature, the ultrasonic shear wave propagates with different velocity and attenuation for each direction of polarization. Ply of a fiber-reinforced layered composite manifest an orthotropic anisotropy with two symmetry axes. Thus shear behavior of each ply is characterized by two shear modulus in planes perpendicular to directions $\mathbf{1}$ and $\mathbf{2}$ (axes represented in Fig. 1), as well as two attenuations for each direction.

As shown in Fig. 1, a shear wave transducer, located at the surface of the composite plate, transmits a shear wave into a layered composite of thickness $d$ and at normal incidence with a polarization angle $\theta$ in the plane $\left[\begin{array}{ll}\mathbf{1} & \mathbf{2}\end{array}\right]$. In pulse echo mode, this transducer receives a reflected wave $u(t)$ by the plate backwall.

Its Fourier transform $U(\omega)=\frac{1}{\sqrt{2 \pi}} \int_{-\infty}^{+\infty} u(t) \exp (-i \omega t) d t$ is expressed in terms of amplitude $U_{\Phi}$ and phase $\Phi$

$$
U\left(\omega, \mathbf{G}_{\mathbf{1 3}}, \mathbf{G}_{\mathbf{2 3}}, \rho, d, \theta, \boldsymbol{\Delta} \alpha, \boldsymbol{\Delta} \theta\right)=U_{\Phi} \exp (i \omega t+i \Phi),
$$

where $\omega$ is the pulsation, $\rho$ the layered composite density. Parameters $\mathbf{G}_{\mathbf{1 3}}, \mathbf{G}_{\mathbf{2 3}}$ are $(1 \mathrm{x} N)$ vectors, $\mathrm{N}$ being the number of composite plies. Each element of 


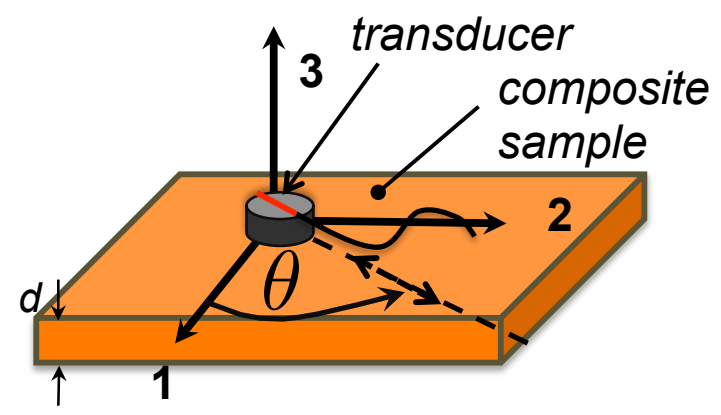

Figure 1: Scheme of the ultrasonic wave measurement set-up in a pulse-echo mode. The transducer emits into the composite sample a shear wave polarized along the direction represented by the line on the transducer (polarization angle $\theta$ with respect to axe $\mathbf{1}$ ). The reflected wave by the backwall is measured by the same transducer at the same polarization angle $\theta$.

$\mathbf{G}_{\mathbf{1 3}}, \mathbf{G}_{\mathbf{2 3}}$ represents for each ply, the shear modulus in planes perpendicular to warp and weft directions, respectively. Variables $\Delta \alpha, \Delta \theta$ are also $(1 \mathrm{x} N)$ vectors whose elements correspond to the ultrasonic attenuation of each layer and the ply orientation according to first layer, respectively.

Following the matrix formalism given in [20, each ply is characterized by a propagator matrix $\mathbf{P}_{n}$,

$$
\begin{aligned}
\mathbf{P}_{n} & =\left(\begin{array}{cc}
P_{n \|} & 0 \\
0 & P_{n \perp}
\end{array}\right) \\
& =\left(\begin{array}{cc}
e^{-j d_{n}\left(\omega \sqrt{\frac{\rho n}{G_{n} \|}}+j \alpha_{n \|}\right)} & 0 \\
0 & e^{-j d_{n}\left(\omega \sqrt{\frac{\rho \rho}{G_{n \perp}}}+j \alpha_{n \perp}\right)}
\end{array}\right)
\end{aligned}
$$

where $\rho_{n}$ and $d_{n}$ are the density and the thickness of the $n^{t h}$ ply, respectively. For this orthotropic material, note that shear moduli in the warp and weft directions, denoted $G_{n \|}$ and $G_{n \perp}$, are related to the elasticity constants $c_{55}$ and $c_{44}$ through following relations $c_{44}=G_{n \| \mid}$ and $c_{55}=G_{n \perp}$ [22]. Due to the anisotropic nature of the material, there are also different attenuations in the warp and weft directions, denoted $\alpha_{n \|}$ and $\alpha_{n \perp}$. In the frequency band 1-5 
$\mathrm{MHz}$, the attenuation is well approximated by a linear law [23,

$$
\alpha_{n \|}=k_{n \|} f \text { and } \alpha_{n \perp}=k_{n \perp} f
$$

where $k_{n \|}$ and $k_{n \perp}$ are the frequency independent attenuation coefficients in the warp and weft direction. The latter attenuation coefficient will be studied for characterizing the composite material.

Normalizing the matrix propagator in 22 by its first element

$$
P_{n 11}=e^{-j d_{n}\left(\omega \sqrt{\frac{\rho n}{G_{n} \|}}+j \alpha_{n \|}\right)}
$$

yields the normalized propagator matrix $\mathbf{P}_{n}^{\prime}$

$$
\begin{aligned}
\mathbf{P}_{n}^{\prime} & =\frac{1}{P_{n 11}} \mathbf{P}_{n} \\
& =\left(\begin{array}{l}
1 \\
0 \quad \exp \left(-j d_{n} \omega\left(\sqrt{\frac{\rho_{n}}{G_{n \perp}}}-\sqrt{\frac{\rho_{n}}{G_{n \| 1}}}+j \frac{\Delta k_{n}}{2 \pi}\right)\right)
\end{array}\right) .
\end{aligned}
$$

The attenuation coefficient difference $\Delta k_{n}$, defined as $\Delta k_{n}=k_{n \|}-k_{n \perp}$, quantifies the dissymmetry between attenuations in the warp and weft directions. Then, an interface matrix $\mathbf{I}_{n, m}$ allows to account for different ply orientations within a layered composite between plies $n$ and $m$ :

$$
\mathbf{I}_{n, m}=\left(\begin{array}{cc}
\cos \left(\Delta \theta_{n, m}\right) & \sin \left(\Delta \theta_{n, m}\right) \\
-\sin \left(\Delta \theta_{n, m}\right) & \cos \left(\Delta \theta_{n, m}\right)
\end{array}\right),
$$

where $\Delta \theta_{n, m}$ represents the orientation difference between plies $n$ and $m$.

Under the assumption of perfect contact between plies and rigid boundaries, the (2x2) transfert matrix $\mathbf{H}$ accounting for the ultrasonic wave propagation within the composite material in a pulse-echo mode is given by

$$
\begin{array}{r}
\mathbf{H}=\prod_{n=1}^{N-1} \mathbf{P}_{n} \mathbf{I}_{n, n+1} \mathbf{P}_{N} \mathbf{I}_{N, N} \mathbf{P}_{N} \prod_{n=N}^{2} \mathbf{I}_{n, n-1} \mathbf{P}_{n-1}, \\
\text { for } N \geq 2,
\end{array}
$$

where $N$ is the number of plies constituting the composite material. From the normalized propagator matrix in Eq. [6], the normalized transfer matrix $\mathbf{H}^{\prime}$ is 
defined as

$$
\begin{array}{r}
\mathbf{H}^{\prime}=\prod_{n=1}^{N-1} \mathbf{P}_{n}^{\prime} \mathbf{I}_{n, n+1} \mathbf{P}_{N}^{\prime} \mathbf{I}_{N, N} \mathbf{P}^{\prime} \prod_{n=N}^{2} \mathbf{I}_{n, n-1} \mathbf{P}^{\prime}{ }_{n-1}, \\
\text { for } N \geq 2 . \\
=\frac{1}{h^{\prime}} \mathbf{H},
\end{array}
$$

where $h^{\prime}$ is the normalizing factor defined as $h^{\prime}=\prod_{n=1}^{N-1} P_{n 11} P_{N 11} P_{N 11} \prod_{n=N}^{2} P_{(n-1) 11}$. For a polarization angle $\theta$ of the transmitted shear wave, components of the reflected shear wave in a pulse-echo mode are expressed as,

$$
\left(\begin{array}{c}
U_{\|} \\
U_{\perp}
\end{array}\right)=\mathbf{I}_{\theta} \mathbf{H} \mathbf{I}_{-\theta}\left(\begin{array}{c}
U_{i n} \\
0
\end{array}\right),
$$

where $U_{i n}$ is the transmitted shear wave and, $U_{\|}$and $U_{\perp}$ are components of the reflected wave with polarization parallel and perpendicular to the receiving transducer, respectively. The interface matrices $\mathbf{I}_{\theta}$ and $\mathbf{I}_{-\theta}$ account for difference of orientation between the transducer polarization and the first ply (weft direction). They are defined as Eq. (7) where $\Delta \theta_{n, m}=\theta$ or $-\theta$. As the transducer is only sensitive to waves with identical polarization as istelf, the measured signal $U(\omega)$ from Eq. (1) corresponds to $U_{\|}$,

$$
\begin{aligned}
U(\omega, \theta) & =U_{\|} \\
& =\mathbf{I}_{\theta} \mathbf{H} \mathbf{I}_{-\theta} U_{i n} .
\end{aligned}
$$

Normalizing the measured signal in $(12)$ by its value at $\theta=0^{\circ}$ yields

$$
\begin{aligned}
\bar{U}(\omega, \theta) & =\frac{U(\omega, \theta)}{U(\omega, 0)} \\
& =\frac{\mathbf{I}_{\theta} \mathbf{H}^{\prime} \mathbf{I}_{-\theta}}{H_{11}^{\prime}} .
\end{aligned}
$$

where $H_{11}^{\prime}$ is the first element of the normalized transfert matrix $\mathbf{H}^{\prime}$ when $\theta=0^{\circ}$. Finally amplitude and phase of the reflected signal are computed from

$$
\begin{aligned}
U_{\Phi}(\omega, \theta) & =|\bar{U}(\omega, \theta)|, \\
\Phi(\omega, \theta) & =\arg (\bar{U}(\omega, \theta)) .
\end{aligned}
$$


Note that for sake of conciseness, dependance of amplitude and phase, $U_{\Phi}$ and $\Phi$, with respect to composite properties are omitted from the notation in above expressions.

In the case of a laminate composite plate with identical elastic properties for each ply, the material is characterized by two out of plane shear moduli, $G_{\perp}$ and $G_{\|}$, in the warp and weft directions of the ply and a single variation of attenuation coefficient $\Delta k$. Considering a constant ply angle orientation, $\Delta \theta$, implementation of expression (13) yields the following simplified expression:

$$
\begin{aligned}
\bar{U}(\omega, \theta) & =\cos ^{2} \Delta \theta\left[\cos ^{2} \theta \cos ^{2}(2 \Delta \theta)+\sin ^{2} \theta\left(\cos ^{2} \Delta \theta e^{-j 2 d \omega \chi}-\sin ^{2} \Delta \theta e^{-j d \omega \chi}\right)\right. \\
& \left.+\frac{\sin (2 \Delta \theta) \sin (2 \theta)}{4}\left(e^{-j \frac{1}{2} d \omega \chi}-e^{-j \frac{3}{2} d \omega \chi}\right)\right], .
\end{aligned}
$$

where $\chi=\sqrt{\frac{\rho}{G_{\perp}}}-\sqrt{\frac{\rho}{G_{\|}}}+j \frac{\Delta k}{2 \pi}$. In that configuration, $d$ refers to the composite plate thickness (and not the ply thickness).

This formulation will be used as a forward modeling for estimating variations of the shear modulus dissymmetry and the attenuation coefficient in order to characterize damage in the case of an unbalanced woven composite plate. The method is presented in the following section.

\subsection{Inversion algorithm}

This study considers laminate plate made of plies with identical orientation and properties. Thus the material is characterized by a single attenuation variation coefficient $\Delta k$ and, by two out of plane shear moduli, in the warp and weft directions, that are denoted $G_{13}=G_{\|}$and $G_{23}=G_{\perp}$. For the considered laminate, the alignement between ply might be not perfect thus misalignment (= orientation variation) between ply is considered through the average orientation $\Delta \theta$. In order to evaluate material damage, the method assesses variations of shear moduli and attenuation dissymmetries between the warp and weft directions. The following parameters are estimated from the shear wave birefringence: the variation of the attenuation coefficient difference $\Delta k$ and shear 
moduli in warp and weft directions in order to quantify the shear modulus ratio variation defined as $R_{g}=\frac{G_{23}}{G_{13}}$.

To characterize the composite material, the inversion algorithm estimates the two shear moduli, the attenuation coefficient difference and the average ply different behavior in the warp and weft directions due to the larger number of fibres in the warp direction. Density of each specimen is $\rho=1819 \mathrm{~kg} / \mathrm{m}^{3}$, and

The received displacement $\bar{U}$ refers to the modeled normalized displacement as defined in Eqs. 13 or 15 according to formalism provided in section 2 Signal $\bar{U}_{O b s}$ refers to experimental reconstructed reflected displacement from measured phase and amplitude signal. The global optimization method "direct search" (patternsearch function from Matlab) is applied on the cost function 16) to find the averaged misalignement $\Delta \theta$, the shear moduli ratio $R_{g}$ from estimation of $G_{13}$ and $G_{23}$ and the attenuation coefficient variation $\Delta k$. The two latter estimated parameters, $R_{g}$ and $\Delta k$, are then used for characterizing the material state. Note that the averaged misalignment is estimated in order to get a better fit between the data and the model but is not used for assessing it in the present paper.

\section{Local damage evaluation on a layered unbalanced woven ply com- posite plate}

\subsection{Description of the layered unbalanced woven ply laminates}

Shear wave birefringence measurements are performed on pristine and damaged layered composite plates. Each composite plate is made of 8 unbalanced woven plies all positioned with the same orientation (meaning that in theory, $\left.\Delta \theta=0^{\circ}\right)$. The material used in this study is a glass reinforced epoxy: the reinforcement is a glass five-harness satin in which $83 \%$ of the fibers ran in the warp direction and $17 \%$ in the weft direction. The unbalanced woven ply shows 


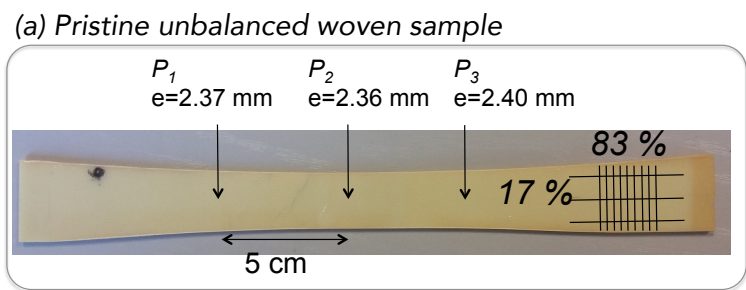

(b) Damaged unbalanced woven sample

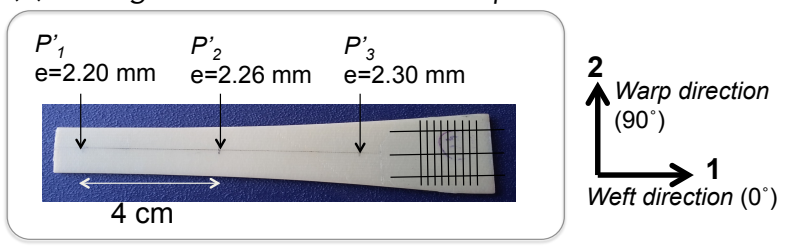

Figure 2: Picture of the (a) pristine and (b) damaged unbalanced composite woven specimen with respective location of the measurement point. The basis is such that shear modulus $G_{13}$ and $G_{23}$ are modulus in weft direction (1) and warp direction (2), respectively.

thickness at different locations on the pristine and damaged specimen is represented in Fig. 2

The damaged sample was obtained from tensile tests with discharge until fracture. Damage behavior study of this composite sample is well detailed in 44. Elastic damage is observed in the warp direction, and elastoplastic damage behavior in the weft direction. Although they are various and complex, main damage processes are fiber-matrix debonding, matrix cracking on the scale of the fibres and small transverse cracks occurring in the material. A loss of stiffness was observed in the sample with a non linear evolution of the damage in the weft direction starting from a loading threshold and reaching a limit corresponding to saturation of the ply with small cracks. Measured damages in the weft and warp directions were around 0.48 and 0.15 just before fracture, respectively. These values were measured on the tensile diagram after the unloading and refer to the loss of stiffness in the Young modulus for each direction $d=1-E / E_{0}$, where $E$ is the damaged material stiffness and $E_{0}$ the initial stiffness (undamaged material).

The ultrasound experimental set-up and processing are now presented. The 

as attenuation coefficient difference between warp and weft direction.

\subsection{Experimental set-up and pre-processing}

Ultrasound measurements are performed at different locations on the specimen as shown in Fig. 2 with a $2.25 \mathrm{MHz}$ piezo-electric shear wave transducer (Panametric V156) in a pulse-echo mode. The polarization angle evolves between $0^{\circ}$ and $360^{\circ}$ with a stepsize of $15^{\circ}$ (see Fig. 3). Generation and reception of impulse signals are monitored with a square wave pulser/receiver (model 5077PR from Sofranel Olympus). And time series are acquired using a Lecroy WS422 oscilloscope. For each set of acquisitions, time series are averaged 30 times at each positions. In order to test robustness and reproducibility of the method, this set of measurements are performed ten times at ten different days for each position and polarization angle. The averaged time series measured on the pristine sample are illustrated in Fig. 4(a) versus the polarization angle. Windowing is applied to the time series to extract the echo from the backwall 10 (see Fig. 4 a with echo between the two dashed lines) and to compute its Fourier transform. Phases and modules at each polarization (Figs. 4a, 4b and 5) are extracted from the frequency domain signal $(0,5-2 \mathrm{MHz})$ and normalized according to their values at polarization angle $\theta=0^{\circ}$. The latter experimental modules and phases, $U_{\Phi, o b s}$ and $\Phi_{o b s}$ are used to reconstruct the displacement $\bar{U}_{\text {Obs }}=U_{\Phi, o b s} \exp \left(i \omega t+i \Phi_{o b s}\right)$. Then minimization of the cost function in (16) is performed to evaluate the attenuation coefficient variation and the shear moduli ratio through estimation of $G_{13}$ and $G_{23}$. The misalignment $\Delta \theta$ is also estimated with average values ranging from $-0.3^{\circ}$ to $1.8^{\circ}$ but is not used for damage characterization.

\subsection{Results}

Birefringence measurement at $1.5 \mathrm{MHz}$ in terms of phase and amplitude are plotted in Fig. 5 for the pristine sample and the damaged sample at 3 different locations (referenced as $P_{1}^{\prime}, P_{2}^{\prime}$ and $P_{3}^{\prime}$ ). Position $P_{3}^{\prime}$ refers to the closest position 


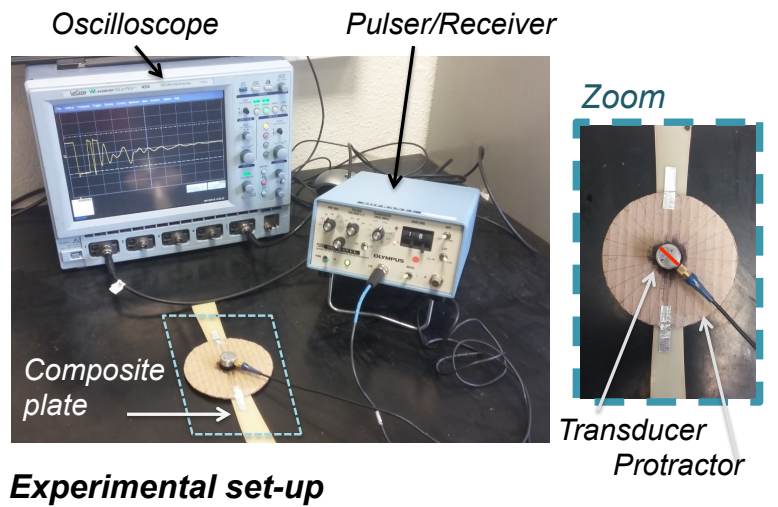

Figure 3: Experimental set-up of the ultrasonic wave measurement. Polarization of the shear wave transmitted by the transducer is represented by a line on the transducer in the zoom picture.

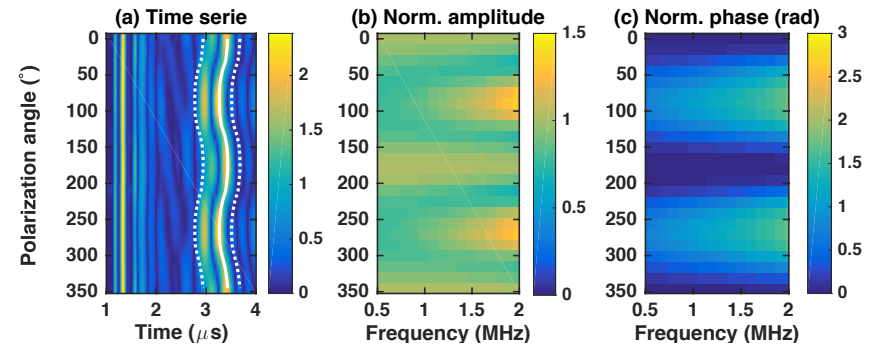

Figure 4: (a) Measured time series (absolute amplitude in $\mathrm{mV}$ ) versus polarisation angle on the pristine composite sample at position $P_{1}$ as shown in Fig. 2 Windowing is applied to extract the backwall echo between the two dashed line and to compute its Fourier transform. (b) Normalized module (no unit) of the first echo in the frequency domain; the amplitude is normalized by amplitude of the module at $\theta=0^{\circ}$. (c)Phase shift (in radian) of the first echo in frequency domain with respect to the phase at polarization angle $\theta=0^{\circ}$. 
to the sample rupture and thus to the most damaged region. Fig. 5 shows that weft direction $\left(0^{\circ}\right)$, damage appears first under the form of micro-cracks along the interface between glass fiber in the warp direction $\left(90^{\circ}\right)$ and the epoxy. As there are $83 \%$ of fiber in the warp direction (compared to $17 \%$ in the weft direction) and due to the direction of sollicitation (tensile stress in the weft $G_{23}$, does not vary significantly, whereas a loss of shear rigidity is observed in 


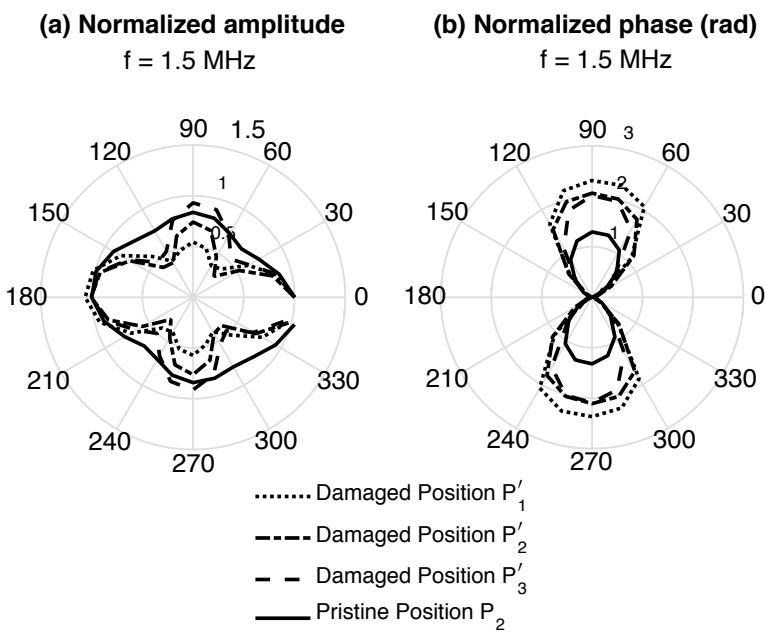

Figure 5: Birefringence measurement at 1.5 MHz for the pristine sample at position $P_{2}$ and the damaged sample at positions $P_{1}^{\prime}, P_{2}^{\prime}$ and $P_{3}^{\prime}$. Averaging is performed over 10 acquisitions. (a) Module of the backwall echo over 10 acquisitions ; the amplitude is normalized by amplitude of the module at $\theta=0^{\circ}$. (b) Phase of the backwall echo over 10 acquisitions; the phase is normalized by the phase at $\theta=0^{\circ}$.

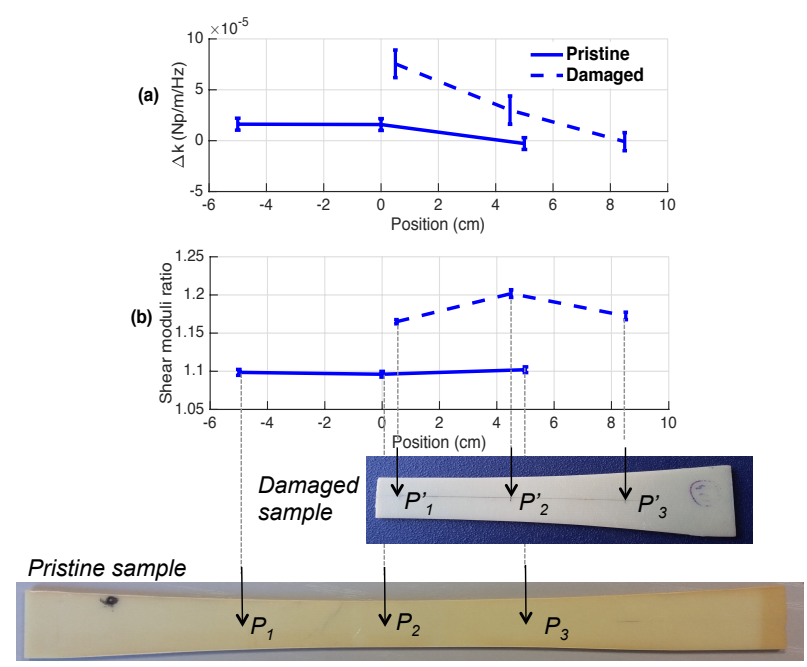

Figure 6: (a) Variation of the attenuation coefficient between warp and weft direction for the pristine sample and the damaged sample versus location of the ultrasound measurement. (b)Ratio of shear wave moduli from warp and weft directions $R_{g}=G_{23} / G_{13}$ for the pristine sample and the damaged sample versus location of the ultrasound measurement. 
the weft direction, that is a decay of shear modulus $G_{13}$ in the weft direction. In overall, this manifests as an increase of the shear moduli ratio $R_{g}$ from about 1.1 on the pristine sample to $1.16-1.2$ for the damage one as seen in Fig. 6 b. Also, as the wave encounters more micro-cracks in the damaged medium, the wave is more attenuated in the warp direction. This results in accentuating the difference of the attenuation coefficient $\Delta k$ between warp and weft directions. Results also shows that attenuation coefficient $\Delta k$ varies with position and decays when getting further from the rupture. In Fig. 6 a, decay of the attenuation coefficient from $75 \mathrm{~Np} / \mathrm{m} / \mathrm{MHz}$ to $-0.9 \mathrm{~Np} / \mathrm{m} / \mathrm{MHz}$ supports the fact that the wave is less attenuated when moving away from the rupture. Larger density of micro-cracks or larger size of micro-cracks can contribute to higher attenuation of the ultrasonic wave. Thus, this result suggests that attenuation is sensitive enough to locally distinct different damage state of the material when moving away from the rupture, probably due to larger density or larger size of microcracks. Ultrasonic attenuation is more sensitive to damage than shear stiffness. This trend is in agreement with results from reference [20] (see Fig. 13 of that reference) where attenuation varies by $75 \%$ as opposed to $20 \%$ for shear stiffness after 5000 cycles of fatigue test on a composite laminate with respect to their initial value.

To summarize, ultrasonic birefringence method is a local method as opposed to the impulse excitation technique that is a global method. The birefringence approach allows to qualify laminate plate damage at a macroscopic scale through 275 estimations of attenuation coefficient variation and the shear moduli ratio. It is complementary to other ultrasound inspection techniques such as acoustic emission. In acoustic emission methods, the major source of acoustic data is the matrix cracking and a minimum sound level from matrix cracking is required. Whereas ultrasonic birefringence allows to measure effects of micro-cracks with any sound level on the shear wave propagation. As acoustic emission, birefringence could be well suitable for continuous monitoring of composite laminates by integrating in situ sensors into the structure as performed with film sensors [24. 


\section{CONCLUSIONS}

285

\section{References}

[1] M. Markham, Measurement of the elastic constants of fibre composites by ultrasonics, Composites 1 (2) (1969) 145-149. 
[10] D. S. Paolino, H. Geng, A. Scattina, A. Tridello, M. P. Cavatorta, G. Belin-
gardi, Damaged composite laminates: Assessment of residual young's mod-

gardi, Damaged composite laminates: Assessment of residual young's modrials, NDT International 5 (1982) $75-86$.

[3] W. Harizi, S. Chaki, G. Bourse, M. Ourak, Mechanical damage characterization of glass fiber-reinforced polymer laminates by ultrasonic maps, Composites Part B: Engineering (2015) 131 - 137.

[4] Y. Thollon, C. Hochard, A general damage model for woven fabric composite laminates up to first failure, Mechanics of Materials 41 (7) (2009) 820-827.

[5] Y. Thollon, Analyse du comportement à rupture de composites stratifiés constitués de plis tissés sous chargements statique et de fatigue, Ph.D. thesis, Aix-Marseille Université, Laboratoire de Mécanique et d'Acoustique de Marseille (2010).

[6] B. Castagnede, J. T. Jenkins, W. Sachse, S. Baste, Optimal determination of the elastic constants of composite materials from ultrasonic wave-speed measurements, Journal of Applied Physics 67 (6) (1990) 2753-2761.

[7] S. Rokhlin, W. Wang, Double through-transmission bulk wave method for ultrasonic phase velocity measurement and determination of elastic constants of composite materials, The Journal of the Acoustical Society of America 91 (6) (1992) 3303-3312.

[8] C. Aristégui, S. Baste, Optimal recovery of the elasticity tensor of general anisotropic materials from ultrasonic velocity data, The Journal of the Acoustical Society of America 101 (2) (1997) 813-833.

[9] A. Castellano, A. Fraddosio, M. Piccioni, Ultrasonic goniometric immersion tests for the characterization of fatigue post-lvi damage induced anisotropy superimposed to the constitutive anisotropy of polymer composites, Composites Part B: Engineering 116 (2017) $122-136$.

[2] I. Scott, C. Scala, A review of non-destructive testing of composite mate- 
ulus through the impulse excitation technique, Composites Part B: Engineering 128 (2017) $76-82$.

[17] A. Arora, Effect of texture and stress on acoustic birefringence, Scripta Metallurgica 18 (8) (1984) $763-766$.

[18] A. Clark, On the use of acoustic birefringence to determine components of plane stress, Ultrasonics 23 (1) (1985) 21 - 30. 
[19] V. Varadan, S. Yang, V. Varadan, Rotation of elastic shear waves in laminated, structurally chiral composites, Journal of Sound and Vibration 159 (3) (1992) $403-420$.

[20] M. Rheinfurth, P. Fey, S. Allinger, G. Busse, Ultrasonic birefringence as a measure of mechanically induced fatigue damage in laminated composites, International Journal of Fatigue 48 (2013) 80-89.

[21] C. Hochard, Optimum design of laminated composite structures, Composite Structures 63 (2004) $159-165$.

[22] S. Rokhlin, D. Chimenti, P. Nagy, Physical ultrasonics of composites, Oxford University Press, 2011, Ch. 1, pp. 18 - 19.

[23] H. C. Kim, J. M. Park, Ultrasonic wave propagation in carbon fibrereinforced plastics, Journal of materials science 22 (1987) 4536 - 4540.

[24] B. Ji-Hun, L. Sung-Woo, C. Seung-Hwan, Characterization of low-velocity impact-induced damages in carbon/epoxy composite laminates using a poly(vinylidene fluoride-trifluoroethylene) film sensor, Composites Part B: 